release and changes in the PNE responsiveness to the TRPA1 channel agonist cinnamaldehyde $(100 \mu \mathrm{M})$.

Results hDPSCs undergo a fibroblastic to neuronal phenotypic switch to PNEs which express the sensory neuronal proteins SP and CGRP. Using qPCR we confirm that PNEs express TLR3, TLR4 and TLR7 mRNA and functional expression of TRPA1 and TRPV1 channels. PNEs pre-treated with PolyI:C $(2 \mu \mathrm{g} / \mathrm{ml})$ for 20 mins generated significantly larger inward $(-10.8773 \mathrm{pA} /$ $\mathrm{pF} ; \mathrm{p}<0.01)$ and outward $(10.0507 \mathrm{pA} / \mathrm{pF} ; \mathrm{p}<0.01)$ currents in response to cinnamaldehyde $(100 \mu \mathrm{M})$ compared to untreated PNEs (-2.347 pA/pF and $2.872 \mathrm{pA} / \mathrm{pF}$ respectively). The electrophysiological events elicited by PolyI:C occurred rapidly, were not sustained and appeared independent of alteration in TRP channel gene expression. PNEs incubated with PolyI:C for $24 \mathrm{~h}$ released significantly greater IL6 $(246.504 \mathrm{pg} / \mathrm{ml} ; \mathrm{p}<0.01)$ and IL8 $(2140.83 \mathrm{pg} / \mathrm{ml} ; \mathrm{p}<0.001)$ levels compared to untreated PNEs.

Conclusion Using a novel human in vitro sensory neuronal model we observed that Poly I:C evoked sensory neuronal hyper-responsiveness with an accompanying pro-inflammatory response. Respiratory viruses may induce similar effects on sensory neurons during exacerbations of airways disease.

\section{S89 HYPERSENSITIVITY TO ADENOSINE TRIPHOSPHATE IN CHRONIC COUGH PATIENTS}

HE Fowles, AH Morice. Hull York Medical School, Hull, East Riding of Yorkshire

\subsection{6/thoraxjnl-2015-207770.95}

Introduction Recent studies have suggested a role for adenosine triphosphate (ATP) activated $\mathrm{P} 2 \times 3$ receptors in the pathophysiology of chronic cough. ATP has previously been used as an inhalational challenge substance in asthmatics and COPD patients, with the main focus being on bronchospasm. We have considered whether chronic cough patients are hypersensitive to inhaled ATP compared to healthy volunteers.

Method The recognised ERS standardised cough challenge using the Ko-Ko digidoser was performed with ATP and AMP as substrates. 20 Healthy volunteers and 20 chronic cough patients were randomised to the order of challenges. C5 (the concentration of substrate causing at least 5 coughs) was compared for ATP and AMP. Average cough response to ATP was compared between the 2 groups.

Results 6 male and 14 female volunteers in each group were randomised to receive cough challenges. Hull Airways Reflux Questionnaire score range was $0-8$ in healthy volunteers and 21-52 in chronic cough patients. 1 healthy volunteer had a mild hypersensitivity reaction to ATP with urticaria. 1 patient withdrew after their first challenge due to worsening cough. No other side effects were reported. 2/19 healthy volunteers coughed with AMP, neither achieved C5. 8/20 chronic cough patients coughed with AMP, 2 achieved C5. 18/20 healthy volunteers coughed with ATP with 15 achieving C5. 19/19 chronic cough patients coughed with ATP, 18 achieved C5. The chronic cough patients had a greater cough response at lower concentrations of ATP as demonstrated in Figure 1.

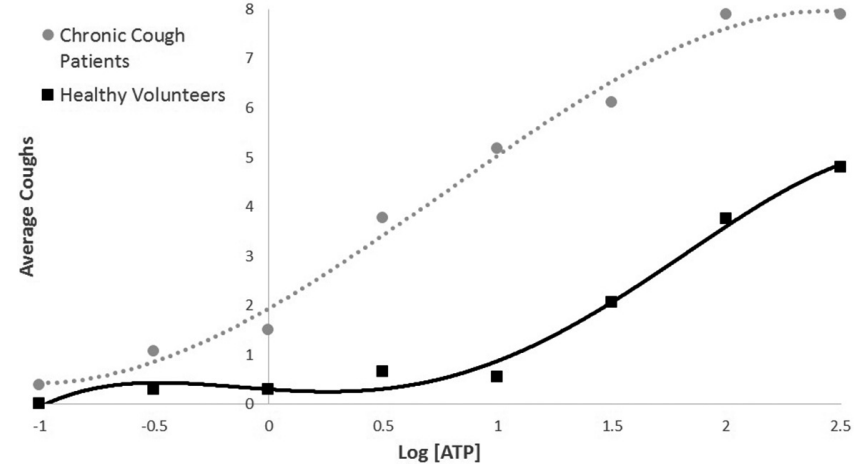

Abstract S89 Figure 1 Average cough response to ATP in healthy volunteers and chronic cough patients

Discussion Previous human ATP challenges have documented cough as a symptom but none have objectively measured the cough response in chronic cough patients. We present here the first results demonstrating that chronic cough patients have increased sensitivity to ATP compared to healthy volunteers. This supports a role for ATP driven receptors in the cough reflex arc and supports further research in this area as a target for treatments in chronic cough.

\section{S90 'CHRONIC COUGH, CAUSE UNKNOWN': A QUALITATIVE STUDY OF PATIENT PERSPECTIVES OF IDIOPATHIC COUGH}

${ }^{1} \mathrm{~K}$ Hulme, ${ }^{1} \mathrm{~S}$ Dogan, ${ }^{1} \mathrm{SM}$ Parker, ${ }^{2} \mathrm{~V}$ Deary. ${ }^{1}$ Respiratory Medicine, North Tyneside Hospital, Northumbria Healthcare NHS Foundation Trust, North Shields, UK; ${ }^{2}$ Northumbria University, Newcastle, UK

\subsection{6/thoraxjnl-2015-207770.96}

Introduction and objectives Idiopathic chronic cough patients have symptoms that persist despite trials of empirical treatment with no underlying cause found. Higher-order brain processes are involved in modulating the cough reflex, but very little is known about the psychological processes underlying idiopathic cough. As the first step in the development of a complex intervention, we sought to elicit an in-depth understanding of patient experience of this condition.

Methods Fourteen patients (12 females, mean age $=59$ years) participated in qualitative interviews theoretically based upon the comprehensive cognitive-behavioural model. Interviews were thematically analysed and cross-validated using the guidelines outlined by Braun and Clark (2006).

Results Eight key themes emerged illustrating the complex, allencompassing nature of idiopathic cough. 'Individual vulnerability' described precipitating factors possibly linked with cough onset. 'More than just a cough' highlighted the co-occurrence of severe physical and emotional experiences. 'Cough in the social sphere' highlighted the effort of dealing with others' reactions and concerns about the contagious image. 'Cough and Identity' described how the cough often defines the person. The occurrence of 'Vicious circles' became apparent, contributing to cough maintenance. 'The battle for control' highlighted the unpredictable nature of the cough, its subsequent impact and the management strategies employed to counter this. Framing the 'Cough in 
relation to other health conditions' provided coughers with a point of reference and some coherence to an otherwise confusing condition. The theme "At the end of the line': the cough healthcare journey' described the care experienced and the continuing search for answers.

Conclusions The onset and persistence of idiopathic cough is complex, involving many interlinking factors. Experiential evidence confirmed previous findings of the involvement of biological (e.g. urge-to-cough sensations) and psychological (e.g. attention) mechanisms. Importantly, it also highlighted the role of the social dimension in how the cough is perceived and managed. These insights suggest a valuable target for future interventions, which accordingly need to take a multi-disciplinary and integrative approach.

\section{REFERENCE}

1 Braun V, Clarke V. Using thematic analysis in psychology. Qual Res Psychol. 2006;3:77-101

\section{S91 A RANDOMISED, DOUBLE-BLIND, PLACEBO- CONTROLLED CROSSOVER STUDY TO ASSESS THE EFFICACY OF A SINGLE DOSE OF 100 MG OF VRP700 BY INHALATION IN REDUCING THE FREQUENCY AND SEVERITY OF COUGH IN ADULT PATIENTS WITH IDIOPATHIC PULMONARY FIBROSIS}

1/ Satia, ${ }^{2} \mathrm{H}$ Badri, ${ }^{2} \mathrm{R}$ Dockry, ${ }^{1} \mathrm{~N}$ Chaudhuri, ${ }^{3} \mathrm{G}$ Brown, ${ }^{3} \mathrm{~K}$ Abbott-Banner ${ }^{2} \mathrm{~A}$ Smith. ${ }^{1}$ University Hospital of South Manchester, Manchester, UK; ${ }^{2}$ University of Manchester, Manchester, UK; ${ }^{3}$ Verona Pharma PIc, London, UK

\subsection{6/thoraxjnl-2015-207770.97}

Background Cough is a common, troublesome symptom in idiopathic pulmonary fibrosis (IPF), but the underlying mechanisms are poorly understood and effective therapies are lacking. VRP700 is thought to inhibit ion-channels found on sensory afferents innervating the airways. We aimed to investigate the efficacy of VRP700 in reducing cough frequency in patients with IPF.

Method A single centre double-blind randomised, placebo controlled crossover study in patients with IPF with chronic cough. Patients were randomised to receive a single inhaled dose of VRP700 (100 mg) or placebo and then crossed-over after a 7 day washout period. The primary endpoint was the number of coughs in the $4 \mathrm{~h}$ following the end of nebulisation for VRP700 compared with placebo, measured using an objective cough monitoring system (VitaloJAK, Vitalograph Ltd). Secondary endpoints included urge to cough visual analogue scale (VAS), cough severity VAS, and dyspnoea VAS, recorded at 1,2 and 4 h postdose, at the end of the day and 24 hrs post-dose.

Results Twenty five patients were screened, 5 were ineligible and therefore 20 were randomised [mean age $69.8( \pm 6.9)$ yrs, 12 female, mean $\mathrm{FEV}_{1} 1.97 \pm 0.39 \mathrm{~L}$, mean FVC $\left.1.86 \pm 0.42 \mathrm{~L}\right]$. The geometric mean number of coughs in the $4 \mathrm{~h}$ following VRP700 treatment was significantly higher compared with placebo [136.8 (95\% CI 80.3- 233.1) vs. 64.9 (95\% CI 38.1110.6), $\mathrm{p}<0.001)$. There was no evidence of an order or period effect. The difference in cough counts was greatest during the first hour after VRP700 nebulization [63.3 (95\% CI 36.4-109.8) vs. 24.1 (95\% CI 13.9-41.9), p < 0.001). For reported cough severity, urge to cough, and dyspnoea severity scores there were no differences between VRP700 and placebo at almost all timepoints, apart from the dyspnoea severity VAS at $24 \mathrm{~h}$ post-dose, where VRP700 was significantly better than placebo $(p=0.012)$.
Conclusion VRP700, administered by nebuliser as a single inhaled dose of $100 \mathrm{mg}$, did not reduced the frequency and severity of cough in IPF patients with troublesome cough. Instead the inhalation of VRP700 seemed to evoke coughing.

\section{Mechanisms of airway inflammation and remodelling}

\section{S92 MATRIX METALLOPROTEINASE-1 ACTIVATION BY MAST CELL TRYPTASE CAUSES AIRWAY REMODELLING AND IS ASSOCIATED WITH BRONCHIAL HYPER- RESPONSIVENESS IN PATIENTS WITH ASTHMA}

${ }^{1} \mathrm{~S}$ Naveed, ${ }^{1} \mathrm{D}$ Clements, ${ }^{2} \mathrm{D}$ Jackson, ${ }^{1} \mathrm{D}$ Shaw, ${ }^{2} \mathrm{~S}$ Johnston, ${ }^{1} \mathrm{SR}$ Johnson. ${ }^{1}$ University of Nottingham, Nottingham, UK; ${ }^{2}$ Imperial College, London, UK

\subsection{6/thoraxjnl-2015-207770.98}

Introduction Matrix Metalloproteinase-1 (MMP-1) is a collagenase, which is present, in its inactive form, in the airways, lung parenchyma and in broncho-alveolar lavage (BAL) fluid of patients with asthma. We hypothesised that MMP-1 could be activated during asthma exacerbations leading to extra-cellular matrix (ECM) processing which contributes to airway remodelling.

Methods Patients with stable, BTS stage $2 / 3$ asthma, and healthy controls underwent Juniper asthma questionnaire, spirometry, methacholine challenge and bronchoscopy. Bronchial washings were processed for MMP-1 protein and activation. A second cohort of 14 patients with mild and 16 with moderate asthma and 10 controls underwent rhinovirus inoculation and had BAL fluid collected 14 days before and 4 days after inoculation. MMP-1 activity was assessed by fluorescent activity assay. ECM was prepared from decellularised airway smooth muscle (ASM) cultures. Cell proliferation was measured by MTT reduction assay and cell counts. Mast cell supernatants were obtained from cultures of HMC-1 cells activated using Phorbol 12-myristate 13-acetate/Calcium ionophore.

Results Pro-MMP-1 was expressed more strongly in bronchial washings in asthma than controls $(\mathrm{P}=0.0003)$. After rhinovirus inoculation, asthma symptoms increased and lung function fell. BAL MMP-1 activity increased in asthma patients compared with controls $(\mathrm{P}=0.047)$. MMP-1 protein and activity was positively associated with fall in $\mathrm{FEV}_{1}(\mathrm{R}$ square $=0.3618)(\mathrm{P}=$ 0.0039 ) post viral inoculation. Activated, but not control, mast cell supernatants increased both expression of pro- and active MMP-1 by ASM cell cultures. This was blocked almost completely by inhibitors of tryptase but not chymase or MMPs. Recombinant tryptase activated MMP-1 in vitro. ECM obtained from both control and asthma derived ASM cells treated with activated mast cell supernatants during matrix synthesis and ECM treated directly after decellularization with active MMP-1 $(10 \mathrm{ng} / \mathrm{ml})$ enhanced subsequent ASM growth by 1.5 fold $(\mathrm{P}<$ $0.05)$.

Conclusions MMP-1 expression and activity in bronchial fluid is enhanced during asthma exacerbations and is associated with increased BHR. MMP-1 activation by mast cell tryptase processes ASM derived ECM to enhance ASM growth in-vitro. Our findings suggest that ASM/mast cell interactions during exacerbations may contribute to airway remodelling by generating a proproliferative matrix. 